\title{
Low stimulus environments: reducing noise levels in continuing care
}

\author{
Juliette Brown, Waleed Fawzi, Amar Shah, Margaret Joyce, Genevieve Holt, Cathy McCarthy, Carmel Stevenson, Rosca Marange, Joy \\ Shakes, Kwesi Solomon-Ayeh \\ East London NHS FT
}

\begin{abstract}
In the low stimulus environment project, we aimed to reduce the levels of intrusive background noise on an older adult mental health ward, combining a very straightforward measure on decibel levels with a downstream measure of reduced distress and agitation as expressed in incidents of violence. This project on reducing background noise levels on older adult wards stemmed from work the team had done on reducing levels of violence and aggression.
\end{abstract}

We approached the problem using quality improvement methods. Reducing harm to patients and staff is a strategic aim of our Trust and in our efforts we were supported by the Trust's extensive programme of quality improvement, including training and support provided by the Institute for Healthcare Improvement and the trust's own Quality Improvement team.

Prior to the project we were running a weekly multi-disciplinary quality improvement group on the ward. We established from this a sub-group to address the specific problem of noise levels and invited carers of people with dementia on our ward to the group. The project was led by nursing staff. We used a noise meter app readily downloadable from the internet to monitor background noise levels on the ward and establish a baseline measure. As a group we used a driver diagram to identify an overall aim and a clear understanding of the major factors that would drive improvements. We also used a staff and carer survey to identify further areas to work on. Change ideas that came from staff and carers included the use of the noise meter to track and report back on noise levels, the use of posters to remind staff about noise levels, the introduction of a visual indication of current noise levels (the Yacker Tracker), the addition of relaxing background music, and adaptations to furniture and environment. We tested many of these over the course of nine months in 2015, using the iterative learning gained from multiple PDSA cycles.

The specific aim was a decrease from above $60 \mathrm{~dB}$ to below $50 \mathrm{~dB}$ in background noise on the wards. Following our interventions, we have managed to decrease noise levels on the ward to $53 \mathrm{~dB}$ on average.

The success of this project to date has relied on the involvement of ward staff and carers - those most affected by the problem - in generating workable local solutions. As many of the change ideas amounted to harm free interventions it was easier for us to make a case to test them out in the real-life setting. Nevertheless we were surprised at how effective such seemingly simple ideas have been in improving the environment on the ward. We have incorporated the change ideas into routine practice and are advising other wards on similar projects.

\section{Problem}

The project was based on a continuing care ward in Hackney, East London, part of East London NHS Foundation Trust, a mental health trust serving a population of over 800,000 in East London. The trust has an improvement aim to provide the highest quality mental health and community care in England by 2020. The ward was involved prior to this project in improvement work to reduce violence and aggression.[1]

The ward is home to ten inpatient beds. It is a continuing care ward for patients with severe dementia which is complicated by challenging behaviour that cannot be managed in other care environments.

The ward is staffed by a multi-disciplinary team comprising mental health nurses, occupational therapists, psychologists, healthcare assistants, and psychiatrists, working closely with community mental health teams and intermediate care teams.
We hypothesised that levels of noise on the ward were a problem that impacted on the quality of life for patients and the quality of care on the ward. Carers and staff confirmed this view.

\section{Background}

Noise is defined by Basner et al (2014) as "unwanted sound"[2] and is a well-recognised problem for patients and staff in hospital environments (Currie, Ruddy, and Mohammed, 2013),[3] with elderly populations considered to be a sub-group particularly 'at risk' (Muzet, 2007).[4]

As is the case for other hospital environments, psychiatry inpatient units expose populations of patients and staff to noise from a number of sources, including patient buzzers; equipment alarms; noise from staff; noise from other patients; telephones; wheels of trolleys and commodes; and doors, drawers, and bin lids (Ulrich et al, 2008).[5] 
There is evidence that noise levels have been increasing in hospitals since the 1960's (Berglund, Lindvall and Schwela, 1999)[6] and the literature suggests exposure to such environmental noise causes physiological changes in humans, including raising systolic and diastolic blood pressure, changes to heart rate, and is associated with release of catecholamine and glucocorticoid stress hormones (Babisch, 2011).[7]

Blomkvist et al (2005)[8] demonstrated increased stress levels in coronary care unit staff, associated with noise levels in the work environment, and concluded that improved acoustic conditions could reduce risks of conflicts, errors, and positively influence the psychosocial environment of healthcare for patients and staff. In spite of the continuing threat that hospital noise levels pose to patient rehabilitation and staff performance (Ulrich et al, 2008), there are few quality improvement studies published that explore how it can be reduced effectively in clinical settings (Royal College of Nursing, 2015).[9]

Specifically for people with dementia, there is a recognition that quality of care is compromised by the profound communication difficulties associated with severe dementia (Sampson et al, 2014).[10] It is likely that exposure to noise on an ongoing basis is a stressor for people with dementia, and compassionate care needs to anticipate needs that people with severe illness cannot communicate.

\section{Baseline measurement}

We elected to use a measure that directly reflected the aim of the project and would not be onerous to collect on a daily basis, allowing us to track changes very easily over time. As a secondary measure on incidents of violence we used the Datix reporting system to establish a baseline measure of violent and aggressive incidents and allow us to easily generate reports on incidents on the ward.

We found a background $\mathrm{dB}$ level on the ward of $62 \mathrm{~dB}$. Before our project began in January 2015 there was an incident of physical violence on the ward every five days.

\section{Design}

The design of the project used the model for improvement, developed by Associates in Process Improvement and the adopted method of the Institute for Healthcare Improvement.[11]

The project lead and sponsor attended Improvement Science in Action training to gain skills, and gain support for the project as it progressed. We set up a regular meeting time and date. We added a discussion on quality improvement tools and methods to staff supervision in order to enable better involvement and ownership of the project by all ward staff. With the input of carers, we developed a driver diagram to display the relevant factors we needed to tackle. We were supported by other team members who had experience in quality improvement from previous projects. We had the support of a project sponsor at senior level who could help to access funding to introduce change ideas and support the project at senior directorate and executive level.

We established a simple method to take observations of $\mathrm{dB}$ levels and set up a team who could collect the data. We meet as a group on a weekly basis to develop and plan tests of change.

\section{Strategy}

The strategy for implementation was based closely on our driver diagram. We were then supported to structure each change idea using a PDSA plan document, in which we established the hypothesis to be tested by the change idea, and our expectations of the test of change. We closely reviewed the data generated by the test - most of it qualitative in the form of verbal feedback from staff on how things were working in practice.

The first change idea we trialled was use of a noise meter itself to monitor $\mathrm{dB}$ levels and report levels back to staff. The idea was based on learning from other projects in the trust in which a feedback loop alone was effective in changing behaviours.[12] The $\mathrm{dB}$ meter app was downloaded onto smartphones and was fun to use, and encouraged staff involvement. Use of the app was standardised and the app was checked internally against other available apps. Times of monitoring and location on the ward were also standardised to ensure we were monitoring the same thing. We chose times and locations of maximal noise such as mealtimes and in shared areas. We measured over a period of five minutes three times a day and recorded an average for each. The noise meter was an excellent first change idea to generate interest in and understanding of the project.

We found that there was modifiable noise - including that of staff and environment - and noise that was inherent to the circumstances and less amenable to change - such as that generated by severely unwell patients. As the numbers of patients on the ward remained stable throughout we did not consider this a confounder in our measurement of noise levels. In most cases patients were too unwell to engage directly in the improvement work, but carers were very actively involved.

Subsequently we developed a poster to remind staff, patients, and carers of the benefit of lower noise levels. These provided an initial visible reminder. As a result of learning from the posters, we were encouraged to try out a further even more effective visual reminder - the Yacker Tracker - a traffic light system that measures the range of $\mathrm{dB}$ levels and can be set to show red, amber, and green based on the desired level. It is unlikely that we would have had the freedom to initiate such an idea without the license of the improvement work being done in the trust. The Yacker Tracker provided a dramatic visual reminder to staff and carers.

We moved subsequently on to adaptations to the environment. The carer most closely involved in the project suggested the use of felt pads on chairs and table legs. She kindly provided the first batch to the ward. The scrapping of furniture legs on the non-carpeted floor had been a major ongoing source of background noise and the felt pads made an immediate difference in reducing noise levels on the 
ward. This success led us to consider other methods of adapting furniture. We trialled a runner on the trolley legs of the tea trolley, which was less effective. We made further plans to use noise absorbing pictures on the wards.

\section{Results}

Our main outcome measure was average $\mathrm{dB}$ levels on the ward, as measured in a standard way three times a day and averaged out. The level reduced during the run of the project fairly quickly and has remained low, from $62 \mathrm{~dB}$ to $53 \mathrm{~dB}$. We had a baseline period for assessment of normal variation and we saw special cause variation by the last week in February 2015.

Due to staff shortages the numbers of days in which measurement took place was reduced toward the end of the period charted. However we found that levels remained low.

At the same time work was ongoing to reduce violence on the ward. We saw violent incidents reduce. Days between incidents increased from an average of one incident every three days to one incident every six days over a period of continuous monitoring from early 2014 to late 2015, with rules for shifting the average based on control chart guidelines. Staff absence rates also reduced by almost $40 \%$ over the same period, based on monthly reports from Human Resources, and plotted monthly, using the same rules for shifting the average. This data was measured continuously over a period of two years from early 2014 onward with each incident plotted, following a drive to record each incident of violence and aggression whether physical or verbal on incident reporting, and is published separately.[1]

See supplementary file: ds6946.pptx - "Average dB levels Jan Sept 2015"

\section{Lessons and limitations}

The lower stimulus wards projects was a spin off from other improvement work done on the same ward on reducing violence and we benefitted from the experience and success of that work in generating momentum and in steering the course of the project. Many of the staff had experienced the impact of improvement work and were enthused to share skills though supervision. The skill mix of the team included nursing staff, OTs, senior sponsor, and carers. All expressed views and perspectives of great value in progressing the project and it was a pleasure to be able to work together with carers on this kind of project.

It is not possible to conclude whether the noise reduction work had an impact directly compared to other measures to reduce violence but it was felt by staff to be part of the overall effect.

We set a specific, measurable, achievable, and realistic aim. We chose an issue of relevance to staff, patients and carers, where we could have an impact without any major risk of harm. We developed a wide range of factors we needed to address and ensured that each were considered sequentially. We had the support of the quality improvement team for methodology and in helping us display data to best effect. We have been able to talk about our work to executive and non-executive directors, and to other trusts and organisations interested in local improvement work. This helps us to appreciate that the ideas generated by frontline staff are welcomed at senior level. We incorporated data measurement in an entertaining way into our daily routines. Having seen the reduction in $\mathrm{db}$ levels on the ward we have incorporated many of the change ideas into practice, and will go on to share ideas and methods with other similar settings.

\section{Conclusion}

The problem identified in this project was the level of background noise on a continuing care dementia ward, much of it stemming from staff activities of various kinds. The problem was important to staff, patients, and carers, with a negative impact on the ward environment and the experience of care for patients and carers. We used improvement methods and resources provided by East London NHS Foundation Trusts' quality improvement programme and benefitted particularly from the involvement of carers in our project team.

We were surprised by how quickly our interventions impacted on levels of noise on the ward. By providing an effective feedback loop, and making minimal changes to the environment and practice of the ward, we saw a significant effect. We are incorporating our change ideas into daily practice and sharing ideas and methods across similar settings.

\section{References}

1 Brown J, Fawzi W, McCarthy C, et al. Safer Wards: reducing violence on older people's mental health wards, BMJ Qual Improv Report 2015; 4:1 u207447.w2977 doi:10.1136/bmjquality.u207447.w2977

2 Basner M, Babisch W, Davis A, et al. Auditory and non-auditory effects of noise on health. The Lancet 2014, 383 pp. 1325-1332.

3 Currie K, Ruddy A, Mohammed T. Improving the Patient Experience: Reducing Avoidable Noise at Night Service Development Project Final Report, NHS Greater Glasgow and Clyde and Glasgow Caledonian University. 2013. [Online] Available at

http://www.researchgate.net/publication/245022634_Improving_the Patient_Experience_Reducing_Avoidable_Noise_at_Night [Last accessed 30 September 2015].

4 Muzet, A. Environmental noise, sleep and health. Sleep Medicine Reviews 2007; [Online] Available at http://waubrafoundation.org.au/wp-content/uploads/2014/02/MuzetA.-Environmental-noise-sleep-and-health.pdf [Last accessed 30 September 2015].

5 Ulrich RS, Zimrig C, Zhu X, et al. A review of the research literature on evidence-based healthcare design (part 1). Health 
Environments Research and Design Journal. 2008. 1(3) pp. 61-125.

6 Berglund B, Lundvall T, Schwela DH. Guidelines for Community Noise. World Health Organisation, Geneva, 1999. [Online] Available at whqlibdoc.who.int/hq/1999/a68672.pdf [Last accessed 30 September 2015].

7 Babisch W. Cardiovascular effects of noise. Noise Health 2011[serial online] 13:201-4. Available at http://www.noiseandhealth.org/text.asp?2011/13/52/201/8014 [Last accessed 30 September 2015].

8 Blomkvist V, Eriksen CA, Theorell T, Ulrich R, Rasmanis G. 'Acoustics and psychosocial environment in intensive coronary care. Occup Environ Med 2005; 62 [Online] Available at http://oem.bmj.com/content/62/3/e1.full [Last accessed 30 September 2015].

9 Royal College of Nursing (2015) Human factors - work environment: noise [Online] Available at http://www.rcn.org.uk/development/practice/patient_safety/human_f actors - work environment/noise [Last accessed 30 September 2015].

10 Sampson EL, White N, Leurent B, et al. Behavioural and psychiatric symptoms in people with dementia admitted to the acute hospital: prospective cohort study. Br J Psychiatry. 2014. September; 205(3): 189-196.

11 Institute for healthcare Improvement Model for Improvement [Online]. Available at http://qi.elft.nhs.uk/model-for-improvement/ [Last accessed 17 December 2015]

12 Cottney A. Using league tables to reduce missed dose medication errors on mental healthcare of older people wards. BMJ Qual Improv Report 2015;4:1 u204237.w3567

doi:10.1136/bmjquality.u204237.w3567

\section{Declaration of interests}

No competing interests.

\section{Acknowledgements}

We would like to thank the staff at East London NHS Trust particularly Maggie Joyce, Mrs McAllister, and the quality improvement team for their contribution to this work.

\section{Ethical approval}

This work met criteria for operational improvement activities exempt from ethics review. 